\title{
Probiotics and prebiotics, targeting obesity with functional foods
}

\author{
Hijova E \\ Institute of Experimental Medicine, Faculty of Medicine, Safarikiensis University, Kosice, Slovakia \\ emilia.hijova@upjs.sk
}

\begin{abstract}
OBJECTIVES: This review aimed to discuss mechanisms through which gut microbiota could act as a key modifier of obesity and related metabolic abnormalities. Obesity is a growing health epidemic with an increasing incidence in European countries. It results from a combination of genetic, psychological, physiological and environmental factors. Probiotic and prebiotic ingredients provide an optimal target for the development of novel functional foods used in the prevention of obesity development as one of risk factors of chronic diseases. Knowledge about prebiotic and probiotic mechanisms of action, and novel functional foods supplemented with probiotics and prebiotics will facilitate their clinical application and development of personalized healthcare strategies (Fig. 1, Ref. 40). Text in PDF www.elis.sk KEY WORDS: gut microbiota, obesity, functional foods, nutrition.
\end{abstract}

\section{Introduction}

Obesity has become one of the largest global health challenges that currently feature in our society. The prevalence of obesity has been continuously rising worldwide such that it is considered an epidemic. According to the World Health Organization (WHO), in $2035,39 \%$ of people in today's society will be affected by obesity (WHO, 2000). International Obesity Task Force (IOTF) suggest that 1.1 billion adults are overweight including 312 million who are obese (James et al, 2004). In parallel to growing waistlines and body mass indices across the globe, modern society is also experiencing a dangerous increase in the prevalence of obesityassociated metabolic derangements.

Obesity is one of the controllable and "modifiable" risk factors of chronic diseases. In-depth research work remains to be done to understand the origins and salient factors promoting obesity and its physiological impacts, such that potential therapeutic avenues for directly targeting metabolic pathology can be developed and effectively implemented. Among such factors, the human gut microbiome has recently taken the center stage bolstered by modern research demonstrating its central role in modulating human health and host metabolism and could act as a key modifier of obesity

Institute of Experimental Medicine, Faculty of Medicine, Safarikiensis University, Kosice, Slovakia

Address for correspondence: E. Hijova, MVDr, PhD, Institute of Experimental Medicine, Faculty of Medicine, LF UPJS, SNP 1, SK-040 11 Kosice, Slovakia.

Phone: +421.55.2343463

Acknowledgements: This publication was supported by the Operational Program Integrated Infrastructure within the project: Functional Foods for Healthy Life with ITMS 2014+313012T288 and Demand-driven research for sustainable and innovative food, Drive4SIFood 313011V336, co-financed by the European Regional Development Fund. and related metabolic complications. The complex ecosystem of the gut microbiota comprises over 1,000 unique bacterial strains. Its prokaryotic population outnumbers the total cells in the human body by an order of magnitude (Dinan and Cryan, 2018). The microbiome can dynamically respond to a variety of internal and external physiological cues while being a metabolic gateway between the outer environment and the host regarding the modulation of inflammation, energy metabolism, and body weight homeostasis. Accordingly, perturbances to the microbiome lead to an unstable state of dysbiosis which is linked to the pathophysiology of various metabolic conditions, including obesity. A multitude of microbial survey studies have attempted to define what constitutes an "obese" microbiome, and pinpoint the specific strains that contribute to the development of obesity and related metabolic symptoms. Based on such investigations, a lean phenotype has become to be largely associated with an increased Bacteriodetes-to-Firmicutes ratio, whereas this taxonomic proportion is inverted in obese individuals (Ley et al, 2006).

The ability of the gut microbiome to interconnect genetics, environment, immune system, and brain implies it could account for variables underlying the development of obesity and its metabolic complications. In this regard, multiple mechanistic axes connecting the gut microbiota to obese pathophysiology are being explored. Early evidence suggests that perturbations to the microbiome in obesity favor increased energy harvest from food, resulting in perturbed nutrient partitioning and development of adiposity (Turnbaugh et al, 2006). This is consistent with the role of the microbiome in regulating fat metabolism, in which bacterial fermentation of non-digestible carbohydrates can lead to excessive production of short-chain fatty acids (SCFAs) and other lipogenic precursors (Nehra et al, 2016). In addition, the dysbiosis in microbiome contributes to the initiation of numerous pro-inflammatory pathways that are a hallmark of obese phenotypes. These include increased 
absorption of bacterial lipopolysaccharide (LPS) expressed on the surface of Gram-negative strains, as well as modulation of gut barrier permeability leading to translocation of bacterial endotoxins into systemic circulation (Khan et al, 2016).

In light of these and other functions, the modulation of gut microbiome through dietary intervention has been proposed as a potential treatment for obesity. Dietary habits are the main contributors to the diversity of the human gut microbiota by altering its composition. Short-term changes in diet profile can shift the gut microbiota. Dietetic intervention could then be a good strategy to treat obesity by reducing the energy intake and potentially modulating gut microbiota to aid weight loss. Based on the fact that gut microbiota clearly differs between obese and lean individuals (Markowiak and Śliźewska, 2017), a selective modulation of gut microbiota using probiotics and/or prebiotics has emerged as a potential therapy for the control of weight gain in both obese and susceptible-to-obesity subjects (Sonnenburg and Bäckhed, 2016; Markowiak and Śliźewska, 2017; Ganjayi et al, 2019).

The goals of this review are to explore the recent evidence elucidating the microbiome's role in the etiology of obesity and discuss the development of functional food solutions in combatting obesity for to be able to capitalize on the benefit of prebiotics and probiotics therapies in optimizing host health.

\section{Obesity and intestinal microbiota}

Obesity is now declared to be a current global epidemic. The pathophysiology of obesity is multifactorial. Its development involves unhealthy life style, neuronal and hormonal mechanisms, as well as genetic and epigenetic factors contributing to the imbalance between energy intake and expenditure (Al-Assal et al, 2018). Obesity is a worldwide epidemiologic syndrome characterized by fat mass accumulation, mainly visceral fat. The prevalence of obesity has doubled in the last three decades. In 2016, according to the WHO, more than 1,900 million (39\%) people aged above 18 years were overweight and 600 million $(13 \%)$ were classified as obese (Bray et al, 2017). Approximately $30 \%$ of all deaths in 2017 in Slovakia were attributed to diet (low fruit and vegetable intake, high consumption of sugar and salt, ubiquitous presence of cheap high-calorie foods), while one in seven adults was obese.

Overweight and obesity are a subject of growing concern also in children. Childhood obesity has grown exponentially over the past 25 years in infants and young children (aged 0-5 years) to 42 million in 2013 and being now the most prevalent nutritional disorder globally among children (Isolauri, 2017). One in six 15-year-old men is suffering from overweight and obesity (OECD, 2019).

Obesity is assessed in practice by body mass index (BMI) or ratio of waist-to-hip circumference. High BMI indexes correlate with diet-related chronic diseases such as hypertension, dyslipidemia, type 2 diabetes, cardiovascular diseases, metabolic syndrome, chronic inflammatory and allergic disorders as well as development of some type of cancers (Isolauri, 2017; Duranti et al, 2017). Energy intake and expenditure are highly interconnected and regulated by complex and coordinated mechanisms that ultimately influence brain stem and hypothalamic, limbic, and other central nervous system centers to regulate food intake and energy spending. Multiple hormones and neuronal circuits appear to control these regulatory processes, including leptin providing feedback from the fat itself, ghrelin secreted by the gastric mucosa, various intestinal peptides, and several appetite-regulating neuropeptides (Maruvada et al, 2017). Genetic factors clearly contribute to the control of the physiologic response to caloric excess and hence to the development and maintenance of obesity, while heritability estimates for the variance in BMI range between $40 \%$ and $70 \%$. The dramatic rise in the prevalence of obesity over the past decades has turned attention towards the environment. Greater control of ambient temperature as a socioeconomic development consequence, increased sedentariness or less physical activity as a result of lifestyle changes, and the ubiquitous presence of cheap high-calorie foods have been implicated as important causal factors. Enhanced consumption of high-fat and high-sugar diet have been shown to change microbial ecology leading to the notion that gut microbiota may function as an environmental factor that results in increased energy harvest and obesity.

The human intestinal microbiota contains more than 40 trillion microorganisms. The dominating bacterial phyla in humans, accounting for $90 \%$ of the gut microbiota, are Firmicutes and Bacteroidetes. There are currently more than 274 genera within the Firmicutes phylum, including Bacillus, Lactobacillus, $M y$ coplasma, and Clostridium. Bacteroidetes include more than 20 genera, of which the most abundant genus in the human gastrointestinal tract is Bacteroides.

Human intestinal microbiota represents a complex ecosystem, consisting of numerous diverse sets of bacteria, viruses, archaea, fungi, protists, nematodes, as well as phages deeply implicated in different functions of host metabolism.

The functions of microbiota are summarized as follows:

- metabolite production (the fermentation of complex carbohydrates results in the production of short-chain fatty acids and other lipogenic precursors that are involved in many cellular processes and metabolic pathways, enhancement of the gut barrier function and regulation of immune system and inflammatory responses); - metabolic organ (with enzymatic properties that enhance or supersede our own, such as the ability to degrade resistant dietary or host-derived glycans that transit the distal gut, regulation of the bile acid metabolism, and induction/protection from metabolic endotoxemia);

- vitamin production (microbiota synthetize essential vitamins $\mathrm{B} 12$ and $\mathrm{K}$ which humans cannot produce, their dysregulation results in metabolic pathologies such as obesity and diabetes mellitus type 2);

- influence on epithelial homeostasis (microbiota promote epithelial integrity by influencing the turnover of epithelial cells and modulating mucus properties;

- development of the immune system (both intestinal mucosal defenses and the systemic immune system are modulated by microbiota, resulting in a greater protection against infections and inflammatory diseases);

- influence on pathogen colonization (microbiota compete with pathogens for attachment sites and nutrients, and they produce 
antimicrobial substances), (Koropatkin et al, 2010; Brusaferro et al, 2018).

The gut microbiota are predominantly involved in the fermentation of indigestible carbohydrates into SCFAs, which have been found to exert multiple effects on energy homeostasis, as well as in a cascade of metabolic functions related to host nutrition and health that are crucial for intestinal health (Hijová and Chmelárová, 2007; Thorburn et al, 2014). The most abundant SCFAs are acetate, butyrate, and propionate, which play an important role as substrate for glucose metabolism. These SCFAs comprise $>95 \%$ of the SCFA content. Several animal and human studies have found increased SCFA fecal concentration in obese as compared to lean individuals, suggesting that increased fecal concentration of SCFAs are associated with obesity (Schwiertz et al, 2010).

Obesity is associated with chronic low-grade inflammation and insulin resistance. White adipose tissue is metabolically the most important adipose tissue playing a central role in the inflammatory state and expressing pro-inflammatory cytokines such as TNF- $\alpha$ and interleukins (IL)-1, IL-6, IL-10, and IL-12. In obesity, there is an increase in cytokine production in the white adipose tissue and infiltration of macrophages, which in turn enhances pro-inflammatory cytokines and subsequently induces insulin resistance. Alteration in the composition of the gut microbiota induced by unhealthy diet is a factor contributing to the onset of chronic low-grade inflammation. These alterations result in increased gut permeability known as gut barrier dysfunction. Gut barrier dysfunction causes low-grade inflammation by either directly translocating Gram-negative intestinal bacteria or increasing lipopolysaccharides (LPS) from the outer membrane of Gram- negative bacteria, which also induces metabolic endotoxemia, and in turn generates low-grade inflammation (DeClercq et al, 2016). The change in the composition of the intestinal microbiota can be caused by a number of factors including changes in diet (Fig. 1).

\section{Prebiotics and probiotics as functional food therapies of obesity}

Historically, prebiotics and probiotics have been well-documented for their positive effects on gastrointestinal health. The term "probiotic" means live microorganisms that, when administered in adequate amounts, confer a health benefit on the host while the term "prebiotic" refers to indigestible food ingredients that are fermented by gut microbiota, serve as a substrate selectively utilized by host microbiota and confer health benefits; both prebiotic and probiotic ingredients provide an optimal target for the development of novel functional foods (Gibson et al, 2017). Although the concept of "functional foods" is not precise or consistently defined, some of its unique characteristics include being a conventional or everyday food that is consumed as part of normal/ usual diet and has a positive effect on target function(s) beyond nutrition value/basic nutrition while potentially reducing the risk of disease, and having authorized and scientifically based health claims (Tufarelli and Laudacio, 2016). Functional foods with the potent physiological benefits of prebiotics and probiotics offer

\section{NON-OBESE}

OBESE
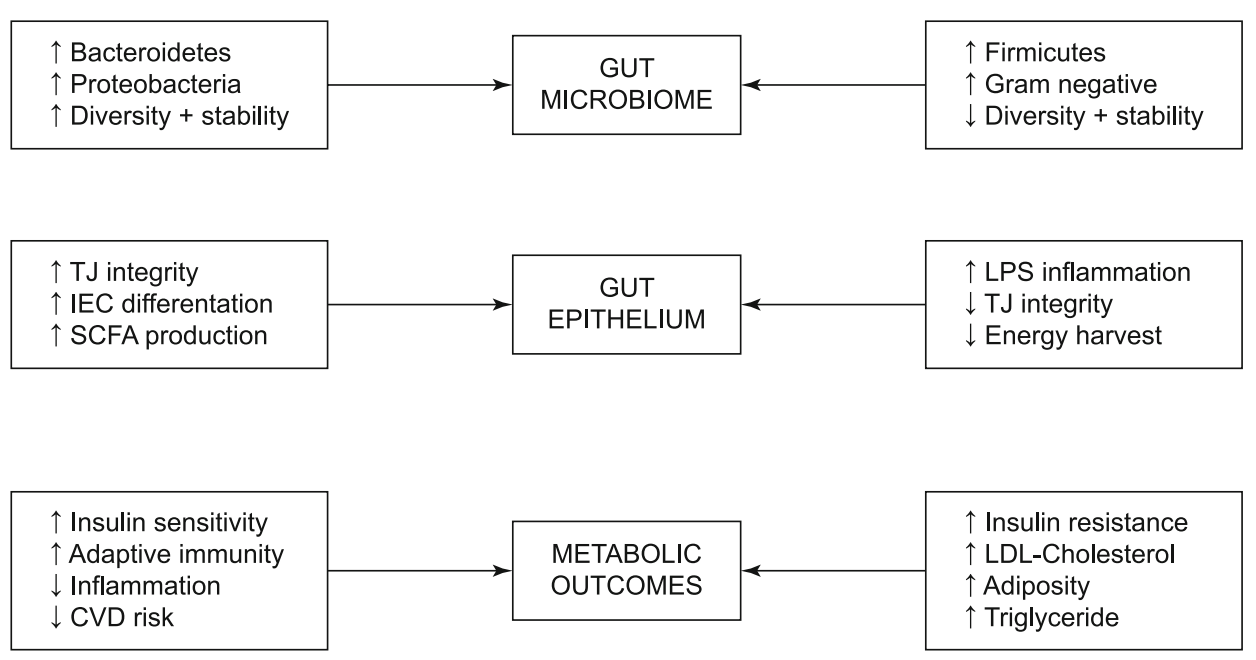

Balanced Microbiome

\section{Dysbiotic Microbiome}

Fig. 1. The change in the composition of the intestinal microbiota can be caused by a number of factors including changes in diet. TJ - Tight Junction; IEC - Intestinal Epithelial Cells; SCFA- Short Chain Fatty Acid; CVD - Cardiovascular Diseases; LPS - Lipopolysaccharide; LDL - Cholesterol - Low Density Lipoprotein Cholesterol 
a unique opportunity for microbiota-targeted dietary modification thrive in the consumer marketplace.

The prebiotics usually include non-digestible, non-hydrolysable carbohydrate forms (i.e., galacto-oligosaccharides (GOSs), fructo-oligosaccharides (FOSs), soybean, oligosaccharides, inulin, cyclodextrins, gluco-oligosaccharides, xylo-oligosaccharides (XOSs), lactulose, lactosucrose, isomaltooligosaccharides, polydextrose) with the ability to reach the distal sections of the human gastrointestinal tract where they are used as nutrients by host intestinal bacteria (Yonis et al, 2015). Non-carbohydrate compounds (such as polyphenol and polyunsaturated fatty acids) are potentially new classes of prebiotics (Gibson et al, 2017).

Dietary sources of prebiotics include asparagus, garlic, leeks, onions, bananas, Jerusalem artichoke, as well as chicory, wheat bran, barley and prebiotic fibers serving as a useful metabolic substrate for intestinal microbiota that convert them via fermentation into SCFAs and other metabolites in the gut lumen. Prebiotic fiber derived from the diet substantiate the growth of known commensal bacterial strains such as Bifidobacterium and various phyla of Lactobacillus, and alters the structure of the gut mucosa, which leads to decreased translocations of bacterial endotoxin and attenuated activation of toll-like receptors (TLRs) at the luminal surface while modulating intestinal $\mathrm{pH}$ prebiotic and creating a bactericidal environment. Obese women treated with inulin/oligofructose prebiotics displayed greater proportions of Bifidobacterium and Faecalibacterium, an effect that coincided with reduced fat mass and serum LPS (Dewulf et al, 2013). Antiobesogenic effects of prebiotics also involve an improvement in glucose and lipid metabolisms as well as in glycemic control. In 2014, Dehghan et al. demonstrated the hypoglycemic and antiinflammatory effects of oligofructose-enriched inulin in patients with type 2 diabetes, where subjects showed marked reductions in fasting glucose, body weight, and inflammatory markers of endotoxemia. Significant decreases in body weight, BMI, and waist circumference have been observed in obese women aged 31-49 years treated with yacon sirup for 17 weeks (Genta et al, 2009), in overweight and obese adults aged 20-70 years treated with oligofructose for 12 weeks (Parnell and Reimer, 2009), and in obese and overweight adults aged 20-50 years treated with rice husk powder/rice bran for 12 weeks (Edrisi et al, 2018). Controversially, oligofructose supplementation combined with healthy lifestyle habits for 12 weeks was not associated with reduced body weight or total body fat (Liber and Szajewska, 2014). The anti-obesogenic role of different strains of Lactobacillus and Bifidobacterium, alone or in combination, as well as Pedicoccus pentosaceus has also been well-established in obese adults and led to reduced weight gain, BMI, waist circumference, and fat mass (Higashikawa et al, 2016; Gomes et al, 2017; Minami et al, 2018; Pedret et al, 2018). Some clinical trials also suggest that the extent of anti-obesogenic effects of probiotics may depend on both the probiotic dose and viable form used. Only after treatment of obese adults aged 20-75 years with a high dose Lactobacillus gasseri BNR17, a reduction in visceral adipose tissue was observed (Kim et al, 2018). It is also important to highlight that healthy non-obese young male adults (18-30 years) can also benefit from probiotic therapy using VSL\#3 in combination with high fat diet in reducing both body weight and fat accumulation (Oesterberg et al, 2015). Mothers 4 weeks before the expected delivery and children up to 6 months of life were supplemented with Lactobacillus rhamnosus GG. In children, the weight gain was lower at the age of 1 and 4 years (Luoto et al, 2010).

The incorporation of prebiotic functional ingredients into foods displays a number of unique physiochemical properties that may potentially:

a) improve the nutrient profile and nutritional value;

b) improve the texture and sensory qualities of foods, primarily due to their bulk, increase the viscosity, and enhance the overall body and mouthfeel;

c) allow reduction in fat, sugar, and energy content without depreciating product taste and texture.

Probiotics have multiple and diverse influences on the host in different ways as follows:

1) antagonistic effects on various microbiota and competitive adherence to the mucosa and epithelium (antimicrobial activity); 2) increased mucus production and enhanced barrier integrity (enhancement of barrier function);

3) modulation of the human immune system (immunomodulation).

Modern marketplace consumers have access to various food products such as fermented products, cheese, and yogurt enriched with probiotic microbiota as functional ingredients targeted to improve digestive health. Their combination (probiotics and prebiotics) termed "synbiotics" may serve to further enhance the beneficial probiotic effects. As compared to those consuming plain yoghurt, obese subjects consuming probiotic-enriched yoghurt (S. thermophiles, and L. bulgaricus as a starter culture, B. lactis Bb12 and prebiotic inulin) significantly reduced their waist circumference and body percentage, as well as lower triglyceride level and increased insulin sensitivity (MohammadiSartang el al, 2018). Probiotics are the potential candidates to be tested in moderate and severe cases of COVID-19 due to several beneficial effects, including easy availability and administration, and safe and unexpensive use. Among patients with underlying comorbid conditions, the gut microbiota has lower diversity which leads to gut dysbiosis. It has been demonstrated that the gut microbiome is altered in cases with COVID-19. Dysregulated inflammation and gut dysbiosis may be the major pathophysiological processes in cases with COVID-19 leading to increased severity of illness and poor clinical outcomes. Therefore, probiotics may act as antiviral agents by interfering with the viral entry into cells and/or inhibit virus replication. Also, with the restoration of the gut and respiratory microbiota, immune function, and gut-lung axis, the course of COVID-19 may be altered (Conte and Toraldo, 2020).

The investigations based on randomized placebo-controlled trials are still needed to implement new prebiotic and/or probiotic treatment as an efficient tool for the prevention and control of obesity and related diseases. This type of therapy has emerged as a unique and exciting opportunity in the management and prevention of obesity and its associated metabolic consequences. 


\section{Conclusion}

The control over the dietary, environmental and lifestyle factors that favor obesity development remain the best solution to the problems related to weight gain. Current literature provides overwhelming evidence for the anti-obesogenic effects of prebiotic and probiotic foods and their potential as a therapeutic avenue for breaching obesity. The focus of work should not be centered only around the physiological effects of probiotic and prebiotic supplementation in combating obesity, but also around the way how functional food delivery vehicles can be optimized to improve microbial viability, stable colonization, and long-term dietary adherence. It is said that obesity is the goal of treatment and diet is the way to achieve the objective. Sometimes it is very difficult and it takes long-term effort to find the right path.

\section{References}

1. Al-Assal K, Martinez AC, Torrinhas RS, Cardinelli C, Waitzberg D. Gut microbiota and obesity. Clin Nutr Exp 2018; 20: 60-64.

2. Bray G, Kim K, Wilding J, Federation WO. Obesity: A chronic relapsing progressive disease process. A position statement of the World Obesity Federation. Obes Rev 2017; 18: 715-723.

3. Brusaferro A, Cozzali R, Orabona C, Biscarini A, Farinelli E, Cavalli E, Grohmann U, Principi N, Esposito $S$. Is it time to use probiotics to prevent or treat obesity? Nutrients 2018; 10: 1613.

4. Conte L, Toraldo DM. Targeting the gut-lung microbiota axis by means of a high-fibre diet and probiotics may have anti-inflammatory effects in COVID-19 infection. Ther Adv Respir Dis 2020; 14: 1-5.

5. De Clercq NC, Groen AK, Romijn JA, Nieuwdorp M. Gut Microbiota in Obesity and Undernutrition. Adv Nutr 2016; 7: 1080-1089.

6. Dehghan P, Pourghassem Gargari B, Asghari Jafar-abadi M. Oligofructose-enriched inulin improves some inflammatory markers and metabolic endotoxemia in women with type 2 diabetes mellitus. A randomized controlled clinical trial. Nutrition 2014; 30: 418-423.

7. Dewulf EM, Cani PD, Clans SP, Fuentes S, Puylaert PGB, Neyrinck AM, Bindels LB, de Vos WM, Gibson GR, Thissen JP, Delzenne N. Insight into the prebiotic concept: Lessons from an exploratory, double blind intervention study with inulin-type fructans in obese women. Gut 2013; 62: 1112-1121.

8. Dinan TG, Cryan JF. Brain-gut-microbiota axis-Mood, metabolism and behaviour. Nat Rev Gastroenterol Hepatol 2017; 14: 69-70.

9. Duranti S, Ferrario C, van Sinderen D, Ventura M, Turroni F. Obesity and microbiota: An example of an intricate relationship. Genes Nutr 2017; 12: 18.

10. Edrisi F, Salehi M, Ahmadi A, Fararoei M, Rusta F, Mahmoodianfard $\mathbf{S}$. Effetcs of supplementation with rice husk powder and rice bran on inflammatory factors in overweight and obese adults following an energy-restricted diet: A randomized controlled trial. Eur J Nutr 2018; 57: 833-843.

11. Ganjayi MS, Balaji M, Sreenivasulu D, Balaji H, Karunakaran RS. Recent Developments in the Prevention of Obesity by Using Microorganisms, Chapter 5, Section 1: Medical Microbiology and Biochemistry, Recent Dev Appl Microbiol Biochem 2019; 47-60.
12. Genta S, Cabrera W, Habib N, Pons J, Carillo IM, Grau A, Sánchez S. Yacon syrup: Beneficial effects on obesity and insulin resistance in humans. Clin Nutr 2009; 28: 182-187.

13. Gibson GR, Hutkins R, Sanders ME, Prescot SL, Reimer RA, Salminen SJ, Scott K, Stanton K, Swanson KS, Cani PD, Verbeke K, Reid G. Expert consensus document: The International Scientific Association for Probiotics and Prebiotics (ISAPP). Consensus statement on the definition and scope of prebiotics. Nat Rev Gastroenterol Hepatol 2017; 14: 491-502.

14. Gomes AC, deSouza RGM, Botello PB, Gomes TLN, Prada PO, Mota JF. The additional effects of a probiotic mix on abdominal adiposity and antioxidant status: A double-blind, randomized trial. Obesity 2017; 25: 30-38.

15. Higashikawa F, Noda M, Awaya T, Danshiitsoodol N, Matoba Y, Kumagai T, Sugiyama M. Antiobesity effect of Pedicoccus pentosaceus LP28 on overweight subjects: A randomized, double-blind, placebo-controlled clinical trial. Eur J Clin Nutr 2016; 70: 582.

16. Hijová E, Chmelárová A. Short chain fatty acids and colonic health. Bratisl Med J 2007; 108: 8, 354-35.

17. Isolauri E. Microbiota and Obesity. In Intestinal Microbiome: Functional Aspects in Health and Disease; Nestle Nutrition Institute Workshop Series; Karger Publishers: Basel, Switzerland 2017; 18: 95-106.

18. James PT, Rigby N, Leach R. The obesity epidemic, metabolic syndrome and future prevention strategies. Eur J Cardiovasc Prev Rehabil 2004; 11: 3-8.

19. Kim J, Yun JM, Kim MK, Kwon O, Cho B. Lactobacillus gasseri BNR 17 supplementation reduces the visceral fat accumulation and waist circumference in obese adults: A randomized, Double-Blind, PlaceboControlled Trial. J Med Food 2018; 21: 454-461.

20. Koropatkin NM, Smith TJ. SusG: A unique cell-membrane-associated $\alpha$-amylase from a prominent human gut symbiont targets complex starch molecules. Structure 2010; 18: 200-215.

21. Khan MJ, Gerasimidis K, Edwards CA, Shaikh MG. Role of Gut Microbiota in the Aetiology of Obesity: Proposed Mechanisms and Review of the Literature. J Obes 2016; 2016: 7353642.

22. Ley RE, Turnbaugh PJ, Klein S, Gordon JI. Human gut microbes associated with obesity. Nature 2006; 444: 1022-1023.

23. Liber A, Szajewska H. Effect of oligofructose supplementation on body weight in overweight and obese children: A randomised, double-blind, placebo-controlled trials. Br J Nutr 2014; 112: 2068-2074.

24. Luoto R, Kalliomäki M, Laitinen K, Isoaluri E. The impact of perinatal probiotic intervention on the development of overweight and obesity. Follow-up study from birth to 10 years. Int J Obes 2010; 34: 1531.

25. Markowiak P, Śliźewska K. Effects of Probiotics, Prebiotics, and Synbiotics on Human Health. Nutrients 2017; 9: 1021.

26. Maruvada P, Leone V, Kaplan LM, Chang EB. The human microbiome and obesity: Moving beyond associations. Cell Host Microbe 2017; 22: 589-59.

27. Minami J, Iwabuchi N, Tanaka M, Yamauchi K, Xiao JZ, Abe F, Sakane N. Effects of Bifidobacterium breve B-3 on body fat reductions in pre-obese adults: A randomized, double-blind, placebo-controlled trial. Biosc Microbiota Food Health 2018; 37: 67-75.

28. Mohammadi-Sartang M, Bellissimo N, Todosy de Zepetnek JO, Brett NR, Mazloomi SM, Fararovie M, Bedeltavana A, Famouri M, Mazloom Z. The effect of daily fortified yoghurt consumption on weight 


\section{$647-652$}

loss in adults with metabolic syndrome: A 10-week randomized controlled trial. Nutr Metab cardiovasc Dis 2018; 28: 565-574.

29. Nehra V, Allen JM, Mailing LJ, Kashyap PC, Woods JA. Gut Microbiota: Modulation of Host Physiology in Obesity. Physiology 2016; 31: $327-335$.

30. OECD/European Observatory on Health Systems and Policies (2019), Slovensko: Zdravotný profil krajiny 2019, State of Health in the EU, OECD Publishing, Paris/European Observatory on Health Systems and Policies, Brusel, 24 p.

31. Oesterberg KL, Boutagy NE, McMillan RP, Stevens JR, Frisard MI, Kavanaugh JW, Davy BM, Davy KP, Hulver MW. Probiotic supplementation attenuates increases in body mass and fat mass during high-fat diet in healthy young adults. Obesity 2015; 23: 2364-2370.

32. Parnell JA, Reimer RA. Weight loss during oligofructose supplementation is associated with decreased ghrelin and increased peptide YY in overweight and obese adults. Am J Clin Nutr 2009; 89: 1751-1759.

33. Pedret A, Valls RM, Calderón-Pérez L, Llauradó E, Companys J, Pla-Pagà L, Moragas A, Martín-Lujás F, Ortega Y, Giralt M, Caimari A, Chenoll E, Genovés S, Martorell P, Codoner FM, Ramón D, Arola L, Solà R. Effects of daily consumption of the probiotic Bifidobacterium animalis subsp. lactis CECT 8145 on antropometric adiposity biomarkers in abdominally obese subject: A randomized controlled trial. Int J Obes 2019; 43: 1863-1868.
34. Schwiertz A, Taras D, Schäfer K, Beijer S, Bos NA, Donus C, Hardt PD. Microbiota and SCFA in lean and overweight healthy subjects. Obesity 2010;18: 190-195.

35. Sonnenburg JL, Bäckhed F. Diet-microbiota interactions as moderators of human metabolism. Nature 2016; 535: 56.

36. Tufarelli V, Laudacio V. An overview on the functional food concept: Prospectives and applied researchers in probiotics, prebiotics and synbiotics. J Exp Biol Agric Sci 2016; 6: 3.

37. Thorburn AN, Macia L, Mackay ChR. Diet, Metabolites, and "Western-Lifestyle“ Inflammatory Diseases. Immunity 2014; 40: 833-842.

38. Turnbaugh PJ, Ley RE, Mahowald MA, Magrini V, Mardis ER, Gordon JI. An obesity-associated gut microbiome with increased capacity for energy harvest. Nature 2006; 444: 1027-1031.

39. World Health Organization. Obesity: Preventing and Managing the Global Epidemic;World Health Organization: Geneva, Switzerland 2000; 5: $252 \mathrm{p}$.

40. Yonis K, Ahmand S, Jahan K. Health benefits and application of prebiotics in foods. J Food Process Technol 2015; 6: 1.

Received February 24, 2021. Accepted March 25, 2021. 